\title{
BMJ Open Effect of different financial competing interest statements on readers' perceptions of clinical educational articles: study protocol for a randomised controlled trial
}

\author{
Sara Schroter, ${ }^{1}$ Julia Pakpoor, ${ }^{1}$ Julie Morris, ${ }^{2}$ Mabel Chew, ${ }^{1}$ Fiona Godlee ${ }^{1}$
}

To cite: Schroter S, Pakpoor J, Morris J, et al. Effect of different financial competing interest statements on readers' perceptions of clinical educational articles: study protocol for a randomised controlled trial. BMJ Open 2016;6:e012677.

doi:10.1136/bmjopen-2016012677

\section{- Prepublication history} and additional material is available. To view please visit the journal (http://dx.doi.org/ 10.1136/bmjopen-2016012677).

Received 16 May 2016 Accepted 18 May 2016

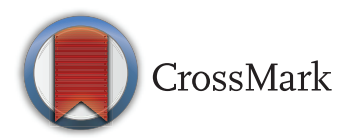

${ }^{1}$ BMJ Editorial, London, UK ${ }^{2}$ University of Manchester, Education \& Research Centre, University Hospital of South Manchester, Wythenshawe Hospital, Manchester, UK

Correspondence to Dr Sara Schroter; sschroter@bmj.com

\begin{abstract}
Introduction: Financial ties with industry are varied and common among academics, doctors and institutions. Clinical educational articles are intended to guide patient care and convey authors' own interpretation of selected data. Author biases in educational articles tend to be less visible to readers compared to those in research papers. Little is known about which types of competing interest statements affect readers' interpretation of the credibility of these articles. This study aims to investigate how different competing interest statements in educational articles affect clinical readers' perceptions of the articles.
\end{abstract}

Methods and analysis: 2040 doctors who are members of the British Medical Association (BMA) and receive a copy of the British Medical Journal (The BMJ) each week will be randomly selected and invited by an email to participate in the study. They will be randomised to receive 1 of 2 Clinical Reviews, each with 1 of 4 possible competing interest statements. Versions of each review will be identical except for permutations of the competing interest statement. Study participants will be asked to read their article and complete an online questionnaire. The questionnaire will ask participants to rate their confidence in the conclusions drawn in the article, the importance of the article, their level of interest in the article and their likeliness to change their practice from the article. Factorial analyses of variance and analyses of covariance will be carried out to assess the impact of the type of competing interest statement and Clinical Review on level of confidence, importance, interest and likeliness to change practice.

Ethics and dissemination: The study protocol, questionnaire and letter of invitation to participants have been reviewed by members of The BMJ's Ethics Committee for ethical concerns. The trial results will be disseminated to participants and published in a peer-reviewed journal.

Trial registration number: NCT02548312; Pre-results.

\section{Strengths and limitations of this study}

- This is the first experimental study to look at the effect of various competing interest statements on readers' perceptions of clinical educational material.

- Financial competing interests are varied. Due to the large sample size required we are only able to evaluate the effect of three financial competing interest statements compared with none.

- We have focused only on financial competing interests. Non-financial interests are also of importance but more difficult to capture and measure.

\section{INTRODUCTION}

Financial ties with industry are common among academics, doctors and institutions and are now sometimes even encouraged, in the spirit of progressive and entrepreneurial academic medicine. ${ }^{1}$ A conflict of interest has been defined as a set of circumstances that creates a risk that professional judgement or actions regarding a primary interest (such as patient welfare or the validity of research) will be unduly influenced by a secondary interest (such as financial gain). ${ }^{12}$

The declaration of competing interests is now the norm for research published in medical journals, with awareness of the potential for such interests to influence findings. ${ }^{3}$ A systematic review of empirical data on the attitudes of academic and clinical researchers toward financial ties revealed concern about the impact of financial competing interests on the choice, conduct and publication of research, however, ties to industry were considered more acceptable when not directly related to the research, disclosure of ties was upfront and study results 
were openly published. ${ }^{4}$ This is supported by work demonstrating lower perceived methodological rigour, credibility and likelihood to act on findings of an industry-initiated trial or a funded trial or where a principal investigator has multiple competing interests. ${ }^{56}$

We previously conducted two randomised controlled trials, which demonstrated that financial competing interests significantly affected readers' perceptions of the credibility of published medical research. ${ }^{7}{ }^{8}$ Chaudhry $e t a l^{7}$ used one research paper and compared the effect of a competing interest statement of "none declared' with a financial statement that the authors were employees of a pharmaceutical company and potentially held stock options in the company. Schroter et $a l^{8}$ used two research papers and three different types of competing interest statement: no competing interests ('none declared'), financial interests (authors were employees of a company and potentially held stock options in the company) and research grant funding (the author was a recipient of funding for studentships and research grants from a company). A third trial, published in the New England Journal of Medicine (NEJM), sent abstracts describing trials of hypothetical drugs to internists and found that respondents assigned less credibility to trials funded by industry, as compared with the same trials reporting National Institutes of Health funding or no funding support. ${ }^{5}$

Less attention has focused on the role of competing interests in clinical education articles, which are intended to guide patient care. These articles do not aim to present raw data, but often use variable and less rigorous methods to search and select from the literature, and comprise the author's own interpretation of such selected literature. Thus, author biases in these articles may be more pervasive yet less visible to general medical readers than those in research papers. Concerned by this, in 2015 the British Medical Journal (The BMJ) introduced a 'zero tolerance' policy for its clinical editorials and some education articles, whereby authors of these articles must not have any relevant financial ties to industry. ${ }^{9}$ In contrast, the NEJM has recently published an editorial by its editor-in-chief and a three-part series questioning the need for stringent policies of competing interests, suggesting that many policies have led to a loss of trust and may 'prevent the dissemination of expertise, thwart productive collaborations, or dissuade patients from taking effective drugs'. ${ }^{10-13}$ This marks a significant departure from the 1990s when the NEJM first introduced a policy to reject anyone with competing interests from writing editorial or review articles. ${ }^{14}$ The argument at the time was that such policies 'do not assume that most physicians or researchers let financial gain influence their judgement. They assume only that it is often difficult if not impossible to distinguish cases in which financial gain does have improper influence from those in which it does not'. ${ }^{15}$

However, little is known about how authors' competing interests affect readers' trust in education articles.
Building on our previous studies using research articles, we will conduct a randomised controlled trial to test the effects of a range of common competing interest statements in educational articles on readers' (practising doctors') interest in an article, their confidence in an article's conclusions, its perceived importance and their likeliness to change practice.

\section{METHODS AND ANALYSIS}

\section{Design and setting}

This will be a randomised controlled trial of practising doctors who are members of the British Medical Association (BMA) and residents in the UK. The BMA has 163 558 UK members, representing $\sim 65 \%$ of all doctors in the UK. This includes 11044 retired doctors and 20889 students who are not practicing. Ninety per cent of all BMA members are sent a copy of the print version of The $B M J$ each week, and all have full online access to the journal (of which $87 \%$ of members have web accounts).

\section{Recruitment and consent}

We will generate a random sample of 2040 BMA members (680 general practitioners (GPs), 680 hospital consultants and 680 junior doctors) and send them a personalised email invitation from The BMJ's editor-in-chief to take part in a research project. We will use an incentive to encourage participation. Recruitment started on 14 November 2015. If we do not receive enough responses, we will email a large random sample of BMA members and ask for volunteers to take part in a research project before assigning them to a group. To avoid biasing participants' responses, details of the study objectives and design will not be given to participants, but we will communicate our findings to participants.

The email invitation will include a link to one of two Clinical Reviews and a link to a short questionnaire on SurveyMonkey. Study participants will be asked to read the article and then complete a short questionnaire. Consent to take part will be assumed by completion of the study questionnaire. Members of The BMJ's Ethics Committee reviewed the study protocol and did not have substantive ethical concerns.

\section{Inclusion criteria and exclusion criteria}

We will include practising doctors in the UK who receive The BMJ and exclude BMA members who have opted out of receiving a free copy of The BMJ, public health doctors, consultant oral/dental surgeons, retired doctors and student members. We will also exclude doctors listed as doing private practice as, due to the way the data about specialty and grade are stored, this is necessary to ensure compliance with our other exclusion criteria.

\section{Intervention}

We will use shortened and modified versions of two Clinical Reviews previously published by The BMJ. These 
are reviews of two conditions commonly seen by doctors, requiring treatment by drugs, and familiar to all clinical specialties. Permission to modify and use the articles has been obtained from the original authors. We will use two Clinical Reviews to help make the findings generalisable beyond a single clinical topic. Each of the two Clinical Reviews will have four permutations differing only in the competing interests statement (from no competing interests to a range of financial interests) for the last of the three authors (box 1). All competing interest statements will appear at the end of the article's main text, just before the references. We will use the same fictional author names and the same fictional institutional affiliations for all versions of the Clinical Reviews. We will use a fictional pharmaceutical company name and use the same company for all permutations with a competing interest. The company name will not be mentioned in the main text of the Clinical Reviews.

\section{Questionnaire}

Participants will be asked to rate on a 10-point Likert scale: how confident they are in the conclusions drawn in the article they have received, how interesting and important they find the article and how likely they are to change their practice on the basis of the article (see online supplementary appendix 1). The question order will be randomised for the first three items (confidence, interest and importance) to reduce question order bias. Before launching the survey, we will pilot it with a convenience sample of doctors to ensure that the instructions are clear and the questions are not ambiguous.

\section{Data collection}

Contact details and demographic information about BMA members will be obtained from the BMA

Box 1 Competing interest statements for the four permutations for each Clinical Review

Authors and institutional affiliations
David Farenden, Resident physician, Department of Medicine,
University of Southaven, Virginia, USA.
Seema Neru, Primary care physician, Noorman Medical Center,
Southaven, Virginia, USA.
Jeremy Barnet, Professor, Institute for Medical Research,
University of Southaven, Virginia, USA.
Competing interests
We have read and understood The BMJ policy on declaration
of interests and declare the following: DF is funded by a NIH
clinician scientist award; SN receives no specific funding; JB
<insert a competing interest statement from A, B, C, D below>:
A. JB has no competing interests.
B. JB has received research funding from Jenka
Pharmaceuticals.
C. JB has received fees from Jenka Pharmaceuticals for consult-
ancies and being an advisory board member.
D. JB has received honoraria and travel expenses from Jenka
Pharmaceuticals for lecturing at a conference.

membership database: name, title, email address, specialty, sex, date of birth and date qualified. All survey data will be gathered electronically using SurveyMonkey. Participants will only be allowed to enter the survey once. Non-responders will be sent email reminders to complete the survey.

\section{Randomisation and blinding}

BMA members eligible for inclusion will be randomised to one of eight groups to receive one of the eight permutations of the Clinical Reviews. The random sample of 2040 BMA members, with one-third taken from GPs, hospital consultants and junior, will be generated from the database of all BMA members by staff at the BMA. The eight permutations of the Clinical Reviews will be randomly assigned a number from 1 to 8 by SS. A computer-generated block randomisation procedure will be used, stratified by type (GPs/hospital consultants/ junior doctors) and gender. Participants will then be randomly allocated to one of the eight groups using random number allocation by JM, who will be blinded as to which group is which. Each group will have equal numbers of GPs, hospital consultants and junior doctors. Participants will be blinded to their group allocation. SS will enrol participants by sending a SurveyMonkey invitation to take part. JM will conduct the statistical analysis blinded to the group allocation; participants will be identified only by study group number, which will not be revealed to JM until after the analysis is complete.

\section{Outcome measures}

\section{Primary outcome measures}

The primary outcome will be the readers' level of confidence in the conclusions drawn in the article, measured on a 10-point Likert scale from 'not at all confident' to 'extremely confident'.

\section{Secondary outcome measures}

The three secondary outcomes will be readers' ratings of the following, all measured on 10-point Likert scales:

1. Importance of the article,

2. Interest in the article,

3. Likeliness to change practice on the basis of the article.

\section{STATISTICAL ANALYSIS AND SAMPLE SIZE RATIONALE} Sample size justification

To have $90 \%$ power to detect a one-unit difference on the 10-point 'confidence' scale between the differing competing interest statement groups, 121 readers are needed in each of the four competing interest statement groups, based on a simple Student's t test with an estimated SD of 2, with a two-sided $1 \%$ significance level to provide some adjustment for multiple testing between the four competing interest statements. However, as differences between the results for the two Clinical Reviews are considered important to quantify, a total of 968 
readers will be required to account for the 8 permutations. Assuming a response rate of around $50 \%$ based on previous The BMJ trials of similar design, at least 1936 readers need to be invited to take part. ${ }^{78}$ Accordingly, for each of the 8 groups, 255 readers ( 85 GPs, 85 consultants and 85 junior doctors) will be invited to take part.

We have assumed that a one-unit difference on the 10-point scale is important on the basis that a 0.5-unit difference was important in our previous studies using a 5-point scale. ${ }^{7} 8$ Similarly, the observed SD for the 5 -point scale was $\sim 1$, and hence we have assumed that, for a 10-point scale, the SD will be twice as large.

\section{Statistical analysis}

Assuming that the data follow a reasonable quasi-normal distribution, a factorial analysis of variance (with competing interest statement and Clinical Review type as the two factors) will be carried out to assess their impact on level of confidence. In addition, the effect of doctor type (GP, consultant or junior doctor), gender, age and the number of years since qualification will be assessed using an appropriate analysis of covariance model. Similar analyses will be carried out for the secondary outcome measures of importance, interest and likeliness to change practice.

\section{Analysis of non-responders}

Non-responders will be compared with responders in terms of age, gender, doctor type (GP, consultant hospital and junior doctor) and number of years since qualification.

\section{POTENTIAL STUDY LIMITATIONS}

This study has potential limitations. Participants will know that they are taking part in a research project and their responses might not reflect the way they typically evaluate an article. They will also be asked to read an article that they might not usually read, which might not be relevant to their practice. However, both articles will be on common conditions, and the task will not require them to have in-depth knowledge of the topic. Response rates for surveys with doctors are often low. ${ }^{16}{ }^{17}$ However, in both of our previous studies, we achieved response rates of $59 \% .^{78}$ This study has a similar sampling frame to the previous studies, but the survey is electronic rather than postal and we do not know if this will influence the response rate or if those who respond will differ from those who do not respond. If the response rate is too low, we will invite a large number of BMA members to volunteer to take part before assigning them to a group. We will exclude doctors doing private practice, so we cannot generalise the results beyond practising National Health Service doctors. However, in an attempt to get a representative sample we will sample doctors from a wide range of clinical specialties.

\section{ETHICS AND DISSEMINATION}

The study proposal and study materials were reviewed by members of The BMJ's Ethics Committee, and they did not have substantive ethical concerns. FG is a member of this committee but was not present at the meeting, where this protocol was discussed. The trial was registered just before recruitment started; any subsequent amendments to the protocol will be uploaded to this registry.

Personal details of BMA members will be treated confidentially. Only SS, JP and JM will have access to the data. JM will receive an anonymised data set.

The results from the trial will be submitted for publication in a peer-reviewed journal. The anonymised individual participant data will be shared on request.

Acknowledgements The authors would like to thank the editors in The BMJ education team (Cath Brizzell, Tony Delamothe, Giselle Jones, Navjoyt Ladher, Emma Parish and Alison Tonks) and Theo Bloom for their help in designing the study; the authors of the original Clinical Reviews for giving them permission to use their work and Emma Parish for editing these articles for use in the study and Keith Bates, Data Analyst at the BMA, for providing data about BMA members and help with sampling.

Contributors FG initiated the study. SS, JP, JM, MC and FG contributed to the study design, including the wording of the competing interests statements, outcome measures and sampling strategy. JM estimated the required sample size and will carry out the statistical analysis. SS and JP will be responsible for running the trial.

Competing interests SS, FG and MC are employed by The BMJ, JP was a Clegg Scholar at The BMJ and JM is a statistics editor for The BMJ.

Ethics approval The study protocol, questionnaire and letter of invitation to participants have been reviewed by members of The BMSs Ethics Committee for ethical concerns.

Provenance and peer review Not commissioned; externally peer reviewed.

Data sharing statement The anonymised individual participant data will be shared on request.

Open Access This is an Open Access article distributed in accordance with the Creative Commons Attribution Non Commercial (CC BY-NC 4.0) license, which permits others to distribute, remix, adapt, build upon this work noncommercially, and license their derivative works on different terms, provided the original work is properly cited and the use is non-commercial. See: http:// creativecommons.org/licenses/by-nc/4.0/

\section{REFERENCES}

1. Lo B, Field MJ, Institute of Medicine (US) Committee on Conflict of Interest in Medical Research, Education, and Practice, eds. Conflict of interest in medical research, education, and practice. National Academies Press, 2009.

2. Thompson DF. Understanding financial conflicts of interest. $N$ Engl J Med 1993;329:573-6.

3. Lundh A, Sismondo S, Lexchin J, et al. Industry sponsorship and research outcome. Cochrane Database Syst Rev 2012;12: MR000033.

4. Glaser BE, Bero LA. Attitudes of academic and clinical researchers toward financial ties in research: a systematic review. Sci Eng Ethics 2005;11:553-73.

5. Kesselheim AS, Roberston CT, Myers JA, et al. A randomized study of how physicians interpret research funding disclosures. $N$ Eng $J$ Med 2012;367:1119-27.

6. Sackett D. Industry-initiated drug trials are far less credible to Canadian internists than investigator-initiated trials. Can J Gen Intern Med 2008;3:29-32.

7. Chaudhry S, Schroter S, Smith $\mathrm{R}$, et al. Does declaration of competing interests affect reader perceptions: a randomised trial? BMJ 2002;325:1391-2.

8. Schroter S, Morris J, Chaudhry S, et al. Does the type of competing interest statement affect reader perceptions of the credibility of research? A randomised trial. BMJ 2004;328 742-3. 
9. Chew M, Brizzell C, Abbasi K, et al. Medical journals and industry ties. BMJ 2014;349:97197.

10. Rosenbaum L. Reconnecting the dots-reinterpreting industryphysician relations. N Engl J Med 2015;372:1860-4.

11. Rosenbaum L. Understanding bias-the case for careful study. N Engl J Med 2015;372:1959-63.

12. Rosenbaum L. Beyond moral outrage-weighing the trade-offs of COI regulation. N Engl J Med 2015;372:2064-8.

13. Drazen JM. Revisiting the commercial-academic interface. N Engl J Med 2015;372:1853-4.
14. Kassirer JP. Tackling conflicts of interest. What the New England Journal of Medicine did. BMJ 2011;343:d5665.

15. Loder E, Brizzell C, Godlee F. Revisiting the commercial-academic interface in medical journals. BMJ 2015;350:h2957.

16. Asch DA, Jedrziewski MK, Christakis NA. Response rates to mail surveys published in medical journals. J Clin Epidemiol 1997;50:1129-36.

17. Cunningham CT, Quan $\mathrm{H}$, Hemmelgarn B, et al. Exploring physician specialist response rates to web-based surveys. BMC Med Res Methodol 2015;15:32. 